\title{
Three dimensions of skew deviation
}

\section{C Brodsky}

\section{Are all skew deviations the clinical expressions of central vestibular dysfunction in three dimensional space?}

S kew means set, placed, or running obliquely; slanting. ${ }^{1}$ For a century, skew deviation has been the descriptive term for an acquired vertical deviation that slants the interpupillary axis to an oblique orientation. ${ }^{2}{ }^{3}$ This condition has traditionally connoted a severe and debilitating neurological injury within the posterior fossa. ${ }^{4}$ Subsequent investigation has refined our understanding of this disorder and expanded the boundaries of this diagnosis. ${ }^{5}$ Working over the past two decades, the promethean team of Brandt and Dieterich have established that skew deviation has a clinical localising value within the posterior fossa ${ }^{6} 7$; that it is a component of the ocular tilt reaction (a triad of skew deviation, binocular torsion, head tilt $)^{7-9}$; and that it is associated with a tilt in the subjective visual vertical. ${ }^{8-11}$ These studies have supplanted our basic descriptive definition of skew deviation with a mechanistic understanding of this complex disorder.

The underlying mechanism for skew deviation involves a unilateral lesion that inhibits (or occasionally stimulates) central otolithic pathways which run from the medulla to the mesencephalon. ${ }^{10}{ }^{11}$ Since a physiological imbalance in central graviceptive tone is normally produced by a head or body tilt in the roll plane, this lesion produces the sensation of body tilt and evokes the same stimulus as is evoked by the Bielschowsky head tilt test. ${ }^{12}$ By activating prenuclear input to the superior rectus and superior oblique muscles of the ipsilateral eye, and the inferior rectus and oblique muscles of the contralateral eye, and by simultaneously inhibiting their antagonists, a cyclovertical divergence is produced, with intorsion of the higher eye, extorsion of the lower eye, and a corrective head tilt toward the side of the lower eye. ${ }^{11}$ This constellation of abnormalities is termed an ocular tilt reaction, which is considered to be compensatory for a tilt in the subjective visual vertical (that is, a perceived tilt of the body relative to the visual environment). ${ }^{8}$ Thus, even with the head held in its tilted position, the patient with an ocular tilt reaction perceives it to be upright. ${ }^{8}$ The beauty of this re-equilibration lies in way that the eyes, the interpupillary axis, and head all roll together to conjointly realign to a new orientation that the central vestibular system erroneously interprets as vertical. ${ }^{8}$ Our internal gyroscope so dictates extraocular muscle and postural control at every level!

But why should the effects of a central vestibular disturbance confine themselves to a single plane in three dimensional space? It turns out that a lesion involving central otolithic pathways unilaterally will alter vertical orientation in the roll plane, while a lesion involving central otolithic pathways bilaterally will alter vertical orientation in the pitch plane (corresponding to forward or backward slant). ${ }^{13}$ And if a roll plane imbalance evokes a cyclovertical divergence of the eyes, what ocular motor signs will a pitch plane imbalance produce? To get an intuitive understanding of the problem, one has only to observe a slanted vertical object held in the sagittal plane. When viewed alternately with either eye, the same object will appear tilted in opposite directions. This exercise shows that the binocular correlate of tilt is slant. ${ }^{14}$ Given that disconjugate visual tilt is perceived as slant, it is not surprising that a bilateral roll plane lesion should alter vertical orientation in the pitch plane.

How the central vestibular system integrates this information is even more fascinating. Brandt and Dieterich have proposed that overlapping pathways modulate roll and pitch function of the vestibulo-ocular reflex. ${ }^{15} \mathrm{~A}$ tone imbalance in the pitch plane indicates bilateral paramedian lesions or bilateral dysfunction of the cerebellar flocculus. ${ }^{15}$ Thus, pathways for roll function can also mediate pitch function if operating in a bilateral mode, making the most efficient use of the vestibular network. The principle behind this operation in physical space resembles the guidance system of an airplane, in which unilateral activation of the brake flap causes the plane to roll, whereas bilateral activation results in downward pitch. ${ }^{15}$ In the same way, a unilateral otolithic imbalance produces an ocular tilt reaction in the roll plane, while a bilateral injury produces a bilateral ocular tilt reaction, in which the torsional components cancel each other out but the vertical components summate. ${ }^{15}$

We have proposed that this summation can produce a bilateral alternating skew deviation, in which patients exhibit an alternating hypertropia of the abducting eye on gaze to either side. ${ }^{16}{ }^{17}$ Because the otolithic pathways corresponding to the anterior and posterior canals are segregated within the brainstem, bilateral lesions tend to selectively affect those otolithic pathways corresponding to one set of canals. ${ }^{16} \mathrm{~A}$ bilateral lesion causing alternating skew deviation may selectively damage the otolithic pathways corresponding to the anterior canals, thereby activating the posterior canal otolithic pathways (which stimulate the superior oblique and inferior rectus muscles bilaterally). ${ }^{16}$ Since the superior oblique muscles are infraductors in adduction and extorters in abduction, there is greater infraduction of the adducting eye, producing a pattern of alternating hypertropia of the abducting eye. ${ }^{16}{ }^{17}$ Conversely, a bilateral injury to otolithic pathways corresponding to the anterior canals would produce alternating hypertropia of the abducting eye. ${ }^{16}$

If skew deviation can reflect a roll or pitch imbalance, can other prenuclear injury to the central vestibular pathways similarly alter horizontal alignment of the eyes? The answer is yes. Acquired comitant esotropia can be produced by structural lesions localised to the posterior fossa such as cerebellar vermal tumours or Arnold Chiari malformations. ${ }^{18-21}$ Since these prenuclear lesions presumably affect central vestibular subserving horizontal rather than vertical vergence, the resulting acquired comitant esotropia can be classified as a horizontal skew deviation. Although these cases do not conform to our original descriptive definition of skew deviation (requiring an oblique or slanted orientation of the visual axes), this nosology recognises a mechanistic overlap with other forms of skew deviation (Table 1).

The physiological underpinnings of horizontal skew deviation may lie in the translational vestibulo-ocular reflex. Primates have developed highly specialised vestibular mechanisms capable of eliciting robust short latency eye movements, known as translational vestibulo-ocular reflexes in response to linear head movements. ${ }^{22-25}$ These reflexes optimise binocular gaze stability during linear disturbances. ${ }^{22}$ In any different heading direction, vestibular signals from the otolith organs of the inner ear encode the direction of the movement and transmit that information to the brainstem, including the motoneurons innervating the eye muscles. ${ }^{22}$ This information is used to generate conjugate version movements (during 
Table 1 The three dimensions of skew deviation

\begin{tabular}{|c|c|c|c|}
\hline Clinical disorder & Plane & Laterality & Pathways \\
\hline Skew deviation & Roll plane & Unilateral & $\begin{array}{l}\text { Brain stem graviceptive pathways } \\
\text { modulating otolithic tone }\end{array}$ \\
\hline $\begin{array}{l}\text { Lateral alternating } \\
\text { skew deviation }\end{array}$ & Pitch plane & Bilateral & $\begin{array}{l}\text { Brain stem graviceptive pathways } \\
\text { modulating otolithic tone }\end{array}$ \\
\hline $\begin{array}{l}\text { Horizontal skew } \\
\text { deviation }\end{array}$ & Surge plane & $?$ & $\begin{array}{l}\text { Cerebellar pathways mediating } \\
\text { translational VOR }\end{array}$ \\
\hline
\end{tabular}

lateral translation) and disconjugate vergence movements (during fore and aft translation) that serve to reduce retinal image slip and provide stable processing of the visual surround. ${ }^{23-25}$

According to Miles, ${ }^{25}$ these vestibularly driven eye movements use radial optic flow and binocular disparity vergence to minimise head movement induced modulation of the fixation plane during translation. Visual tracking mechanisms that address translational disturbances are most accurately tuned for headings in the fore-aft axis ${ }^{22}$ (in the surge plane). Disconjugate vergence movements during fore and aft translations exhibit much higher gains (at or above unity) than conjugate version movements during lateral translation in the lateral plane (approximately $0.5)$, suggesting that the translational vestibulo-ocular reflex is tuned to stabilise binocular gaze in the same depth plane during translatory movements. ${ }^{22}$ Such a vestibularly driven stabilisation of the fixation plane operates at frequencies higher than those of disparity driven vergence eye movements and could be important for the maintenance of stereovision..$^{22}$ The high gain of the vergence response could be related to the low error threshold for stereovision, which is of the order of seconds of arc. ${ }^{22}$ In this way, the translational vestibuloocular reflex more closely resembles the smooth pursuit system (which stabilises images on the fovea without regard to peripheral vision) than the rotational vestibulo-ocular response (which stabilises images only on the peripheral retina). ${ }^{22}$

While prenuclear lesions that disrupt vertical vergence localise to the brain stem, those that disrupt horizontal vergence tend to localise to the cerebellum. Although little is known about the neural substrate of the translational vestibulo-ocular reflex, monkeys with lesions involving the dorsal cerebellar vermis develop an esotropia that is greater at distance than near fixation. ${ }^{26} 27$ The dorsal vermis projects to the fastigial oculomotor region, which has been implicated in the control of vergence. ${ }^{27}$ Neurons have been identified within the cerebellar vermis that modulate with convergence alone or with convergence and saccades. ${ }^{28}$ Humans with global cerebellar dysfunction can have an esophoria during monocular viewing and a smaller esotropia during binocular viewing, suggesting that increased in esotonus to the extraocular muscles with a relatively preserved ability to use horizontal disparity cues to drive motor fusion. ${ }^{29}$ There are theoretical reasons why the cerebellar flocculus may also influence horizontal eye position. In rabbits, the cerebellar flocculus has an inhibitory projection to the medial rectus but not the lateral rectus muscles, so that a lesion in the flocculus could lead to a convergence bias. ${ }^{30}$ Some cells in the flocculus discharge in relation to the angle of vergence. ${ }^{31}$ Nevertheless, the selective ocular motor effects of bilateral flocculectomy and paraflocculectomy on horizontal alignment havenot been defined.

Future study of the perceptual correlates of bilateral alternating skew deviation and acquired comitant esotropia should help to determine whether these vertical and horizontal vergence abnormalities indeed reflect central disturbances in pitch and surge, and whether all skew deviations are the clinical expressions of central vestibular dysfunction in three dimensional space.

\section{Br J Ophthalmol 2003:87:1440-1441}

\section{Author's affiliation}

M C Brodsky, Department of Ophthalmology, University of Arkansas for Medical Sciences, Little Rock, Arkansas, and Arkansas Children's Hospital, 800 Marshall, Little Rock, AR 72202, USA; brodskymichael_@uams.edu

Supported in part by a grant from Research to Prevent Blindness, Inc.

\section{REFERENCES}

1 Webster's ninth new collegiate dictionary. Springfield, MA: Merriam-Webster, 1988:1104.

2 Cogan DG, Thomas CC. Neurology of the Ocular Muscles, 2nd ed. Springfield, IL, 1958:134-5.

3 Smith JL, David NJ, Klintworth G. Skew deviation Neurology 1964;14:96-105.

4 Keane JR. Ocular skew deviation: an analysis of 100 cases. Arch Neurol 1975:32:185-90.

5 Leigh RJ, Zee DS. The neurology of eye movements, 3rd ed. Oxford: Oxford University Press, 1999:463-65

6 Brandt TH, Dieterich M. Different types of skew deviation. J Neurol Neurosurg Psychiatry 1991;54:549-50
7 Brandt T, Dieterich M. Skew deviation with different types of ocular torsion: a vestibular brainstem sign of topographic diagnostic value. Ann Neurol 1993:33:528-34.

8 Brandt T, Dieterich M. Pathologic eye-head coordination in roll: tonic ocular tilt reaction in mesencephalic and medullary lesions. Brain 1987; 110:649-66.

9 Dieterich M, Brandt T. Ocular torsion and tilt of subjective visual vertical are sensitive brainstem signs. Ann Neurol 1993;33:293-9.

10 Halmagyi GM, Brandt Th, Dieterich $M$, et al. Tonic contraversive ocular tilt reaction due to unilaeral mesodiencephalic lesion. Neurology 1990;40:1503.

11 Brandt T, Dieterich M. Vestibular syndromes in the roll plane: topographic diagnosis from brainstem to cortex. Ann Neurol 1994;36:337-47.

12 Kushner BK. Ocular torsion (or rotations around the why axis). J AAPOS 2003; (in press).

13 Brodsky MC. Do you really need your oblique muscles: Adaptations and exaptations. Arch Ophthalmol 2002;120:820-8.

14 Moster ML, Schatz NJ, Bosley TM, et al. Alternating ipsilateral hypertropia on lateral gaze (bilateral abducting hypertropia). Ann Neurol 188;23:190-2.

15 Brandt T, Dieterich M. Central vestibular syndromes in roll, pitch, and yaw planes. Neuroophthalmol 1995; 15:291-301.

16 Brodsky MC, Donahue SP. Primary oblique muscle overaction: the brain throws a wild pitch. Arch Ophthalmol 2001;119:1307-14.

17 Donahue SP, Brodsky MC. Posterior canal predominance in bilateral skew deviation. Br J Ophthalmol 2001;85:1395.

18 Williams AS, Hoyt CS. Acute comitant esotropia in children with brain tumors. Arch Ophthalmol 1987; 107:376.

19 Hoyt CS, Good WV. Acute comitant esotropia: when is it a sign of serious neurological disease. $\mathrm{Br} J$ Ophthalmol 1995;79:408-501.

20 Lewis AR, Kline LB, Sharpe JA. Acquired esotropia due to Arnold-Chiari malformation. J Neuro-ophthalmol 1996;16:49-54.

21 Hoyt CS, Fredrick DR. Serious neurologic disease presenting as comitant esotropia. In: Rosenbaum AL, Santiago AP, eds. Clinical strabismus management. principles and surgical techniques. Philadelphia: WB Saunders, 1999: 152-8.

22 Angelaki DE, Hess BJM. Direction of heading and vestibular control of binocular eye movements. Vis Res 2001:41:3215-28.

23 Paige GD, Tomko DL. Eye movement responses to linear head motion in the squirrel monkey. II. Visual-vestibular interactions and kinematic considerations. J Neurophysiol 1991:65:1183-96.

24 Tomko DL, Paige GD Linear vestibuloocular reflex during motion along axes between nasoocciptal and interaural. Ann NY Acad Sci 1992:656:233-41.

25 Miles FA. The neural processing of 3-D visual information: evidence from eye movements. Eur J Neurosci 1998;10:811-22.

26 Takagi M, Zee DS, Tamargo RJ, et al. Effect of dorsal cerebellar vermal lesions on binocular eye movement control. Invest Ophthalmol Vis Sci 1997;38:S653.

27 Takagi M, Tamargo R, Zee DS. Effects of lesions of the cerebellar oculomotor vermis on eye movements in primate: binocular control. Progr Brain Res 2003;42:19-33.

28 Zhang HY, Gamlin PDR. Single-unit activity within the posterior fastigial nucleus during vergence and accommodation in the alert primate. Soc Neurosci Abstr 1996:22.

29 Versino M, Hurko O, Zee DS. Disorders of binocular control of eye movements in patients with cerebellar dysfunction. Brain 1996:119:1933-40.

30 Ito M, Nisimaru N, Yamamoto M. Specific patterns of neuronal connexions involved in the control of the rabbit's vestibulo-ocular reflexes by the cerebellar flocculus. J Physiol 1977;265:833-54.

31 Miles FA, Fuller JH, Braitman DJ, et al. Long-term adaptive changes in primate vestibulo-ocular reflex. III. Electrophysiological observations in flocculus of normal monkeys. J Neurophysiol 1980;43:1437-76. 